\title{
Numerical and experimental study of strata behavior and land subsidence in an underground coal gasification project
}

\author{
N. N. Sirdesai ${ }^{1}$, R. Singh ${ }^{1}$, T. N. Singh ${ }^{1}$, and P. G. Ranjith ${ }^{2}$ \\ ${ }^{1}$ Department of Earth Sciences, Indian Institute of Technology Bombay, Mumbai, India \\ ${ }^{2}$ Deep Earth Energy Lab, Monash University, Clayton, VIC, 3800, Australia \\ Correspondence to: N. N. Sirdesai (nikhil.sirdesai@gmail.com) \\ Published: 12 November 2015
}

\begin{abstract}
Underground Coal Gasification, with enhanced knowledge of hydrogeological, geomechanical and environmental aspects, can be an alternative technique to exploit the existing unmineable reserves of coal. During the gasification process, petro-physical and geomechanical properties undergo a drastic change due to heating to elevated temperatures. These changes, caused due to the thermal anisotropy of various minerals, result in the generation of thermal stresses; thereby developing new fracture pattern. These fractures cause the overhead rock strata to cave and fill the gasification chamber thereby causing subsidence. The degree of subsidence, change in fluid transport and geomechanical properties of the rock strata, in and around the subsidence zone, can affect the groundwater flow. This study aims to predict the thermo-geomechanical response of the strata during UCG. Petro-physical and geomechanical properties are incorporated in the numerical modelling software COMSOL Multiphysics and an analytical strength model is developed to validate and further study the mechanical response and heat conduction of the host rock around the gasification chamber. Once the problems are investigated and solved, the enhanced efficiency and the economic exploitation of gasification process would help meet country's energy demand.
\end{abstract}

\section{Introduction}

Most of the energy requirement is fulfilled by conventional fuels such as coal, oil and gas with a minor share contributed by nuclear fuel and unconventional energy sources such as solar, wind, tidal and geothermal energy. It has become imperative to address the growing energy demand by using unconventional energy sources and by increasing the efficiency of the conventional sources with the application of novel techniques of fuel recovery. One such novel technique is Underground Coal Gasification (UCG). This technique involves the conversion of coal into fuel gas, also known as syngas, within the earth. The coal undergoes partial combustion by reacting with the mixture of air and steam, which is injected into the seam through one well. The product of the combustion is syngas, which is recovered through the production well/s. The result of the UCG process is a large cavity which is left behind upon the burning of the coal seam.
Due to the combustion of coal, the temperatures inside the reactor/cavity reach up to $1200^{\circ} \mathrm{C}$ (Tian et al., 2011). The rocks around the cavity get heated for a duration of several months. The continuous exposure at such high temperatures for elongated periods of time causes melting, which induces several changes in the physical, mineral and mechanical properties of rocks (Kratzsch, 1983). A huge amount of laboratory research has been carried out to ascertain the effects of high temperatures on the physical (Wu and Liu, 2008; You and Kang 2009; Tian et al., 2012) and the mechanical (Rao et al., 2007; Vishal et al., 2011; Ranjith et al., 2012; Tian et al., 2015) properties of rock. The microstructure of the rock undergoes significant change, thereby affecting the physical and the mineralogical properties (Somerton 1992; Hajpál and Török, 2004). The constituent materials of the rock experience thermal expansion which leads to the creation of new micro fractures and the widening of the inherent micro-cracks (Den'gina et al., 1994). The results ob- 
tained from these tests indicate that heating, effects change in the physical and the thermomechanical properties. However, the effect varies with the change in rock type and the conditions prior to thermal treatment. The change in properties due to heating and the extent of the cavity induces instability in the overlying layer, tending it to fail and fill in the cavity (Kratzsch, 1983; Reddish and Whittaker, 1989). This heavy movement of the overlying area results in subsidence. Cracks formed from the burnt coal seam to the overlying strata, may result in a leak and cause groundwater contamination (Whittaker and Reddish, 1989; Burton et al., 2007). The scale of a commercialized UCG operation will be bigger than that of the pilot and field tests. This would result into notable ground subsidence caused by the large underground cavities. Success of a UCG project depends upon the amount of associated ground movement, thus making it an important factor to evaluate.

The shape of the UCG cavity governs the mechanical stability, the extent of ground subsidence and the economic feasibility of the operation (Shirsat and Riggs 1986; Luo et al., 2009). During UCG, the cavity formed underground spreads out both radially and axially along the gasification channel (Cena et al., 1987). The shape of the cavity depends on the type of UCG process used. As seen in Figs. 1 and 2, the cavity is the largest around the vicinity of the injection well in Linked Vertical Well (LVW) method (Shirsat, 1989). Whereas in the Controlled Retraction Injection Point (CRIP) method, due to constant retraction of injection point, the cavity has a constant cross section (Bell et al., 2011). The CRIP chamber created is basically not more than $20 \mathrm{~m}$ in width and $20 \mathrm{~m}$ in length (Tian, 2013).

In this research programme, a numerical model of the overlying strata was simulated in COMSOL Multiphysics in order to study the behaviour of mechanical parameters. The dimensions and the depth at which the cavity exists were altered to study the strata behavior and to analyse the subsidence caused on the surface. The aim of the ongoing research at the Department of Earth Sciences, Indian Institute of Technology Bombay, is to add to the available pool of scientific knowledge regarding subsidence during a UCG project and to propose possible mitigation techniques so as to minimize the damage caused to human life, infrastructure and economy.

\section{Numerical modelling}

In order to understand the effect of UCG on the overlying strata, a numerical model was prepared using the Finite Element Analysis (FEA) simulation software COMSOL Multiphysics. The process of UCG has been simulated for three coal seam condition. Shallow seated, medium seated and deep seated coal seams have been simulated at a depth of 100,500 and $1000 \mathrm{~m}$ respectively (Fig. 3). This study would help us in determining the effect of cavity depth on subsi-

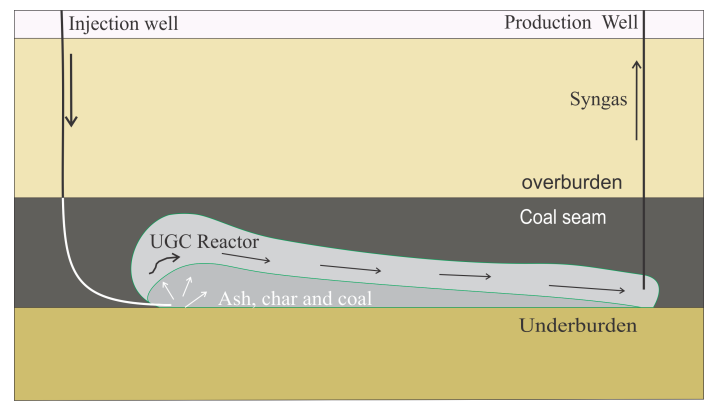

Figure 1. Linked Vertical Well Method (Modified after Shirsat, 1989).

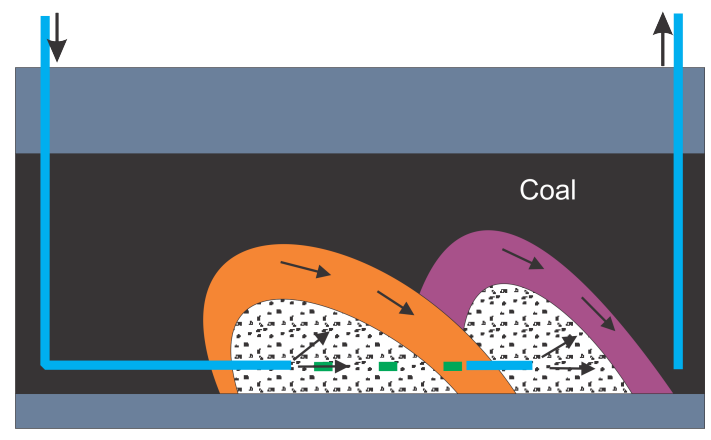

Figure 2. Controlled Retraction Injection Point Method (Modified after Bell et al., 2011).

dence. Similarly, the effect of the cavity width on subsidence has been determined by simulating widths ranging from 20 to $200 \mathrm{~m}$ (Fig. 4). As stated earlier, the cavity created does not exceed $20 \mathrm{~m}$ in width. But, in the multiple inlets and single outlet method, the size of the cavity near the injection points would be large.

The cavity has been visualized to be seated in a host consisting of fine grained sandstone. The mechanical properties of the host rock are shown in Table 1. The host rock is considered to be homogenous in nature. The limitation is to simulate geological discontinuities in the modelling. Thus, the Mohr-Coulomb Failure Criterion has been used. The criterion states that failure occurs when the shear stress and the normal stress acting on any element in the material satisfy the equation

$|\tau|+\sigma \cdot \tan \varphi-c=0$

here, " $\tau$ " is the shear stress, $c$ the cohesion, and " $\phi$ " denotes the angle of internal friction.

With the help of Mohr's circle, this criterion can be written as

$\frac{1}{2}\left(\sigma_{1}-\sigma_{3}\right)+\frac{1}{2}\left(\sigma_{1}+\sigma_{3}\right) \cdot \sin \varphi-c \cdot \cos \varphi=0$.

Initially, the model was simulated without the presence of cavity and gravity was applied to the entire system. Later 

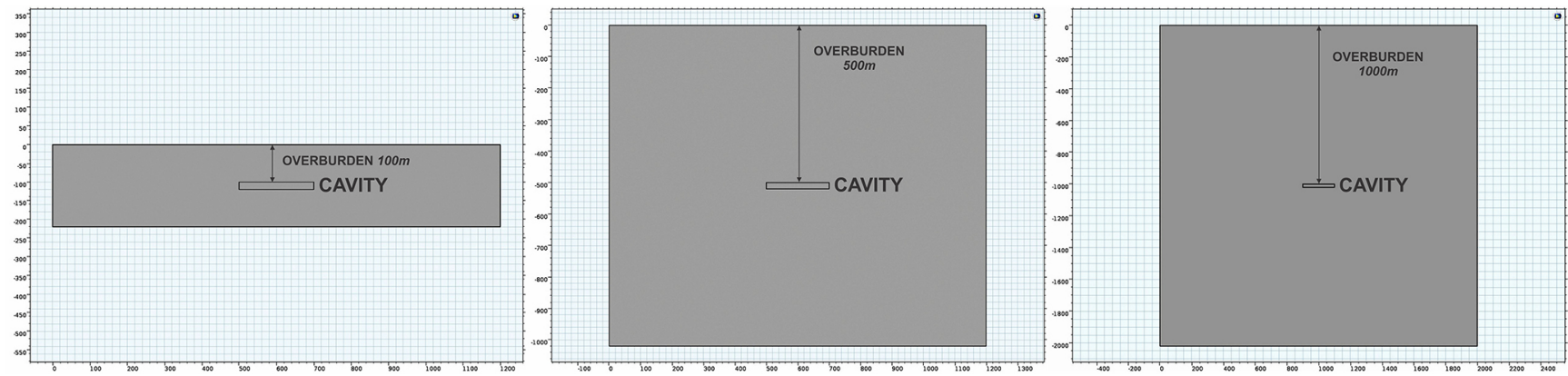

Figure 3. COMSOL Models for Shallow (100 m), Medium (500 m) and Deep (1000 m) seated cavity.

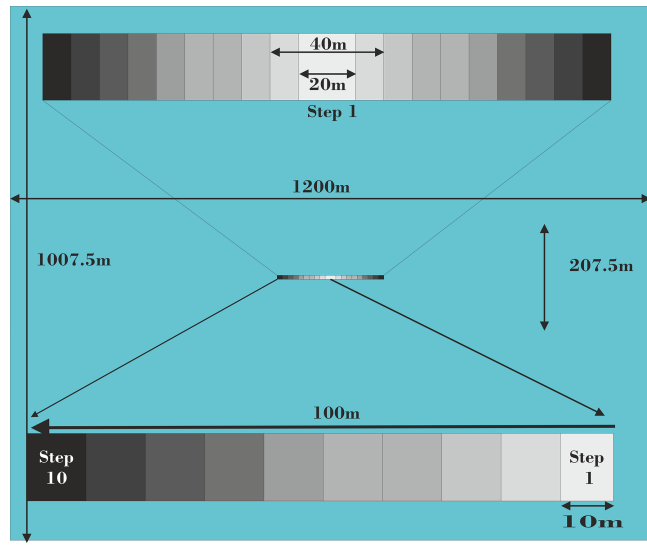

Figure 4. Simulated cavity widths.

Table 1. Mechanical Property of Host Rock.

\begin{tabular}{ll}
\hline Mechanical Property & Value \\
\hline Density & $\left.2231.04\left[\mathrm{~kg}^{-3}\right)\right]$ \\
Young's Modulus & $25.45 \times 10^{9}[\mathrm{~Pa}]$ \\
Poisson's Ratio & 0.24 \\
Cohesion & $10 \times 10^{6}[\mathrm{~Pa}]$ \\
Angle of Internal Friction & $50.19[\mathrm{deg}]$ \\
Dilation Angle & $0[\mathrm{deg}]$ \\
\hline
\end{tabular}

the cavity was modelled and the vertical and the horizontal movement of the upper boundary was observed to study the displacement caused at the surface. Parametric sweep was applied to observe the effect of various cavity widths on the behaviour of strata.

The effect of high temperatures on the strata and the rate of heat transfer was observed by applying high temperature element $\left(1000^{\circ} \mathrm{C}\right)$ to the cavity boundaries. The thermal properties of the host rock are shown in Table 2. Equation (3) was used to calculate the heat transfer through solids.

$\rho \cdot C_{\mathrm{p}} \cdot \frac{\partial T}{\partial t}+\rho \cdot C_{\mathrm{p}} \cdot u \cdot \nabla T+\nabla \cdot q=Q$.
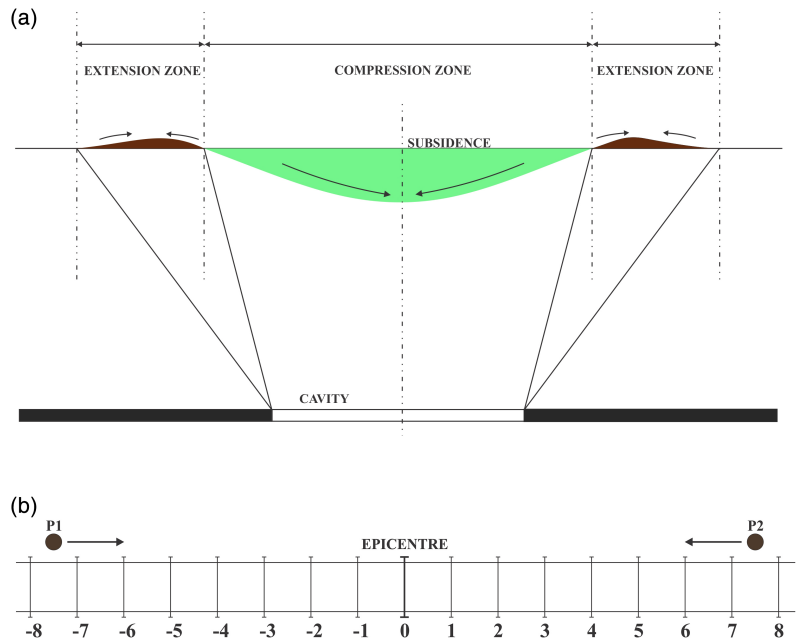

(c)

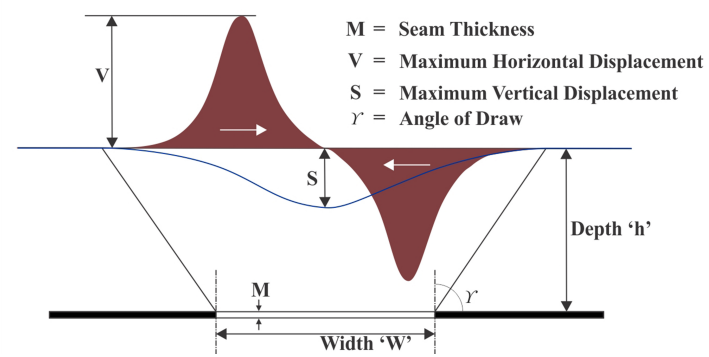

Figure 5. (a) Compression and Extension zones during Subsidence (NCB, 1975; Whittaker and Reddish, 1989). (b) Movement of materials around the epicentre of subsidence. (c) Empirical Subsidence Model (NCB, 1975; Whittaker and Reddish, 1989).

For a steady-state problem the temperature does not change with time and the first term disappears. The equation includes the following material properties: density " $\rho$ ", heat capacity " $C_{\mathrm{p}}$ ", velocity field " $u$ " and a heat source (or sink) " $Q$ ". 

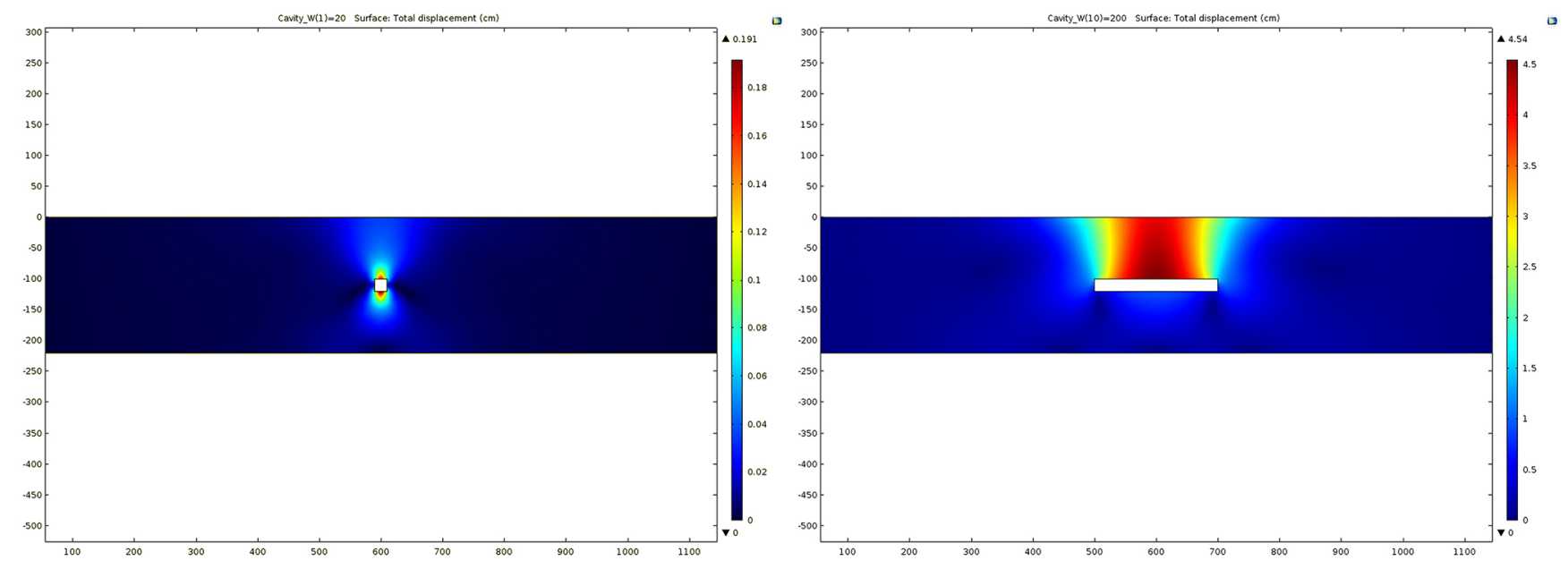

Figure 6. Total strata displacement around a shallow seated cavity.
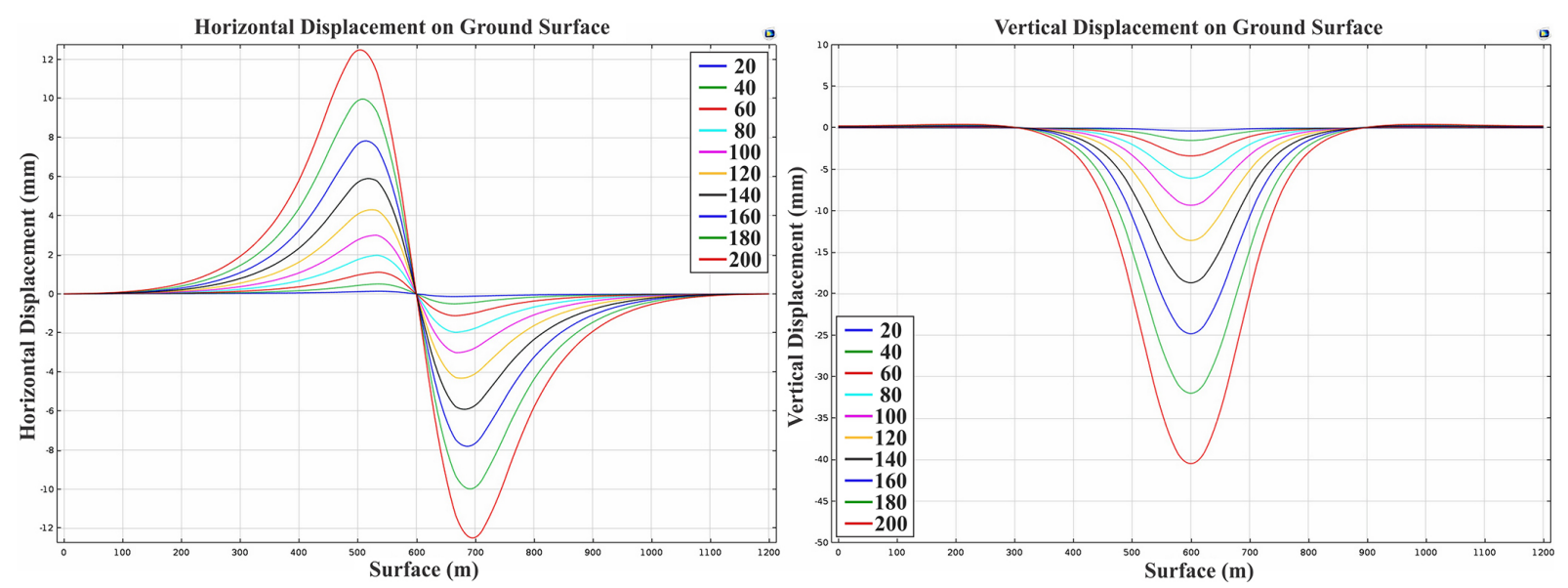

Figure 7. Horizontal and Vertical displacement of surface induced by a shallow seated cavity.

Table 2. Thermal Property of Host Rock.

\begin{tabular}{ll}
\hline Thermal Property & Value \\
\hline Thermal Conductivity & $2.348\left[\mathrm{~W}(\mathrm{~m} \cdot \mathrm{K})^{-1}\right]$ \\
Heat Capacity at Constant Pressure & $752.72\left[\mathrm{~J}(\mathrm{~kg} \cdot \mathrm{K})^{-1}\right]$ \\
Coefficient of Thermal Expansion & $11 \times 10^{-6}\left[\mathrm{~K}^{-1}\right]$ \\
\hline
\end{tabular}

\section{Results and discussion}

During the period of 1950-1965, 200 coalfields were observed for subsidence and an empirical model was formed. The model includes the change in surface profile, zones of extensions and compression and the direction of ground movement (NCB, 1975; Whittaker and Reddish, 1989). A schematic view of the subsidence model is provided in Figs. 5a and 5c. The subsided surface is categorized in two zones namely, Extension Zone and Compression Zone. Sub- sidence causes the material, near the subsided zone, to move towards the epicentre, thereby creating a compression zone. As the material moves away from the sides, an extension zone develops (Fig. 5a). If the epicentre is considered to be the origin i.e. " 0 ", the material on the negative $x$ axis move towards the origin, as seen in Fig. 5b. Due the surficial movement, particle P1 is drawn towards the epicentre. This causes its value to increase. Similarly, particle P2 is also drawn towards the centre, thereby causing a decrease in its value. If the movement of all such particles are plotted, the resulting graph of the total horizontal displacement would be as seen in Fig. 5c.

Figure 6 shows the total displacement caused on the surface due to the smallest and the largest cavity. The $20 \mathrm{~m}$ wide cavity does not affect the overlying strata as much as the $200 \mathrm{~m}$ wide cavity. A cavity induces instability in the overlying strata. This affects the equilibrium of the rock directly above the cavity causing it to break away and fill in the cavity. This process continues until an equilibrium is attained. 

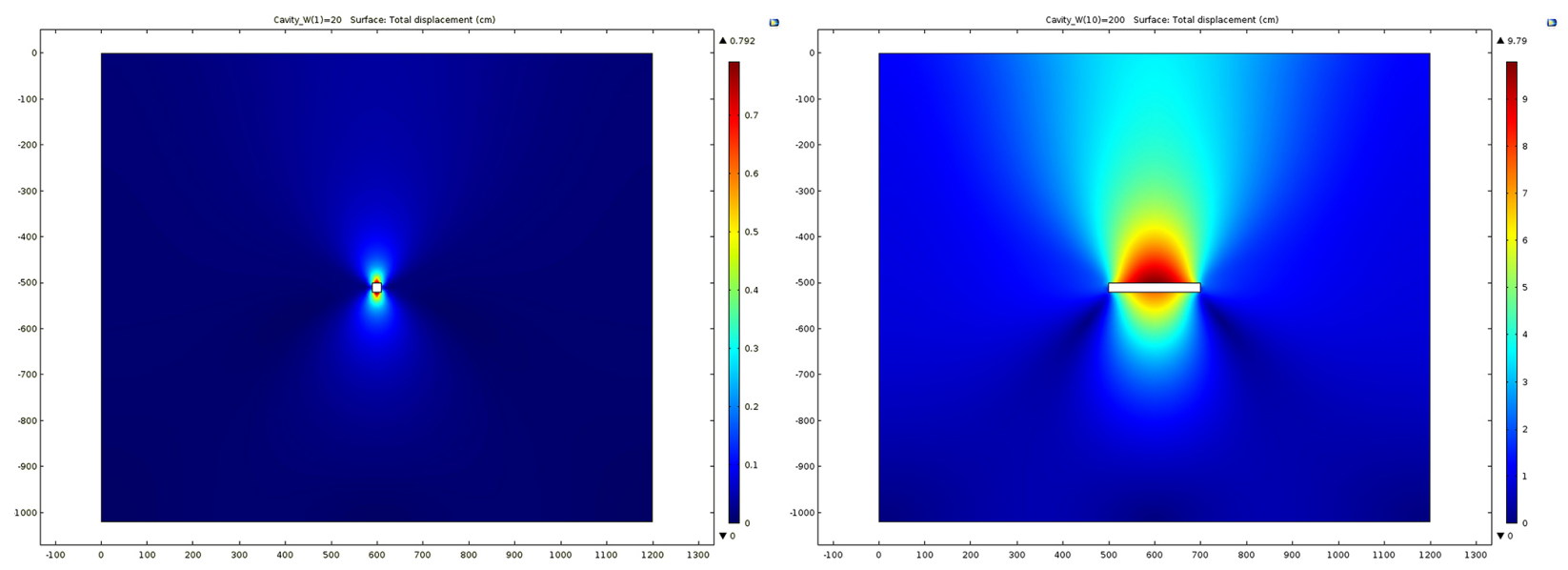

Figure 8. Total strata displacement around a medium seated cavity.
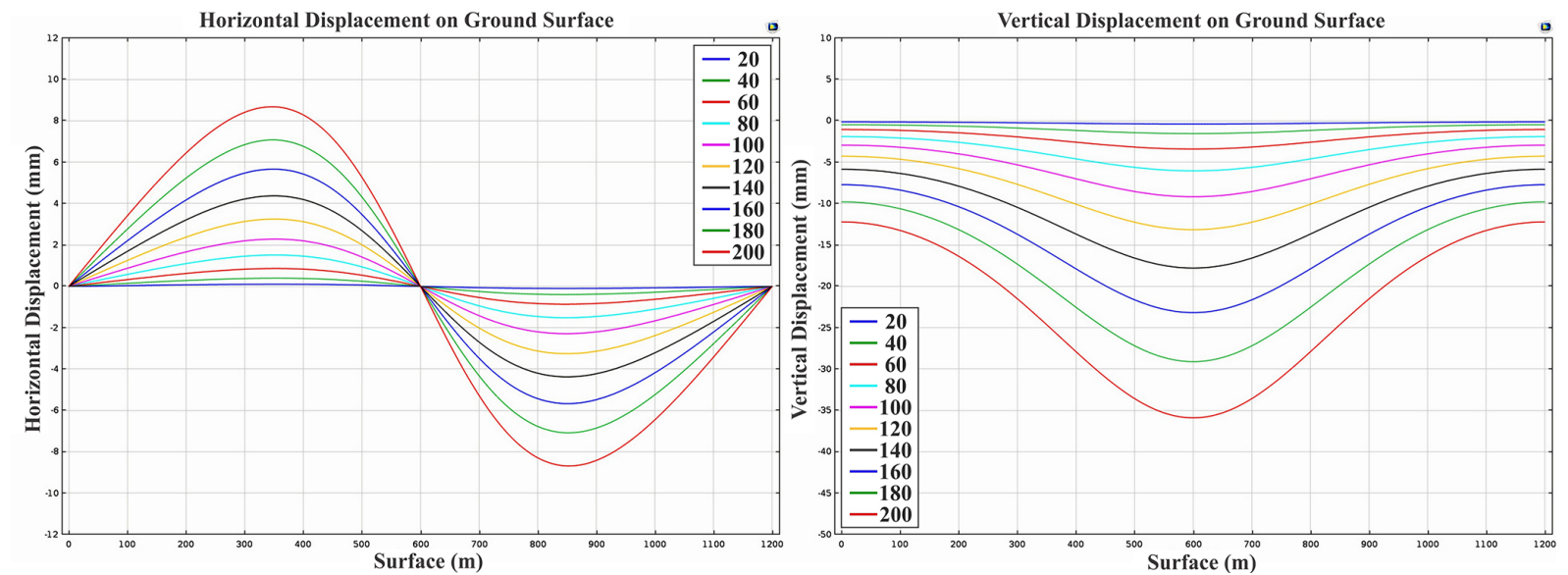

Figure 9. Horizontal and Vertical displacement of surface induced by a medium seated cavity.
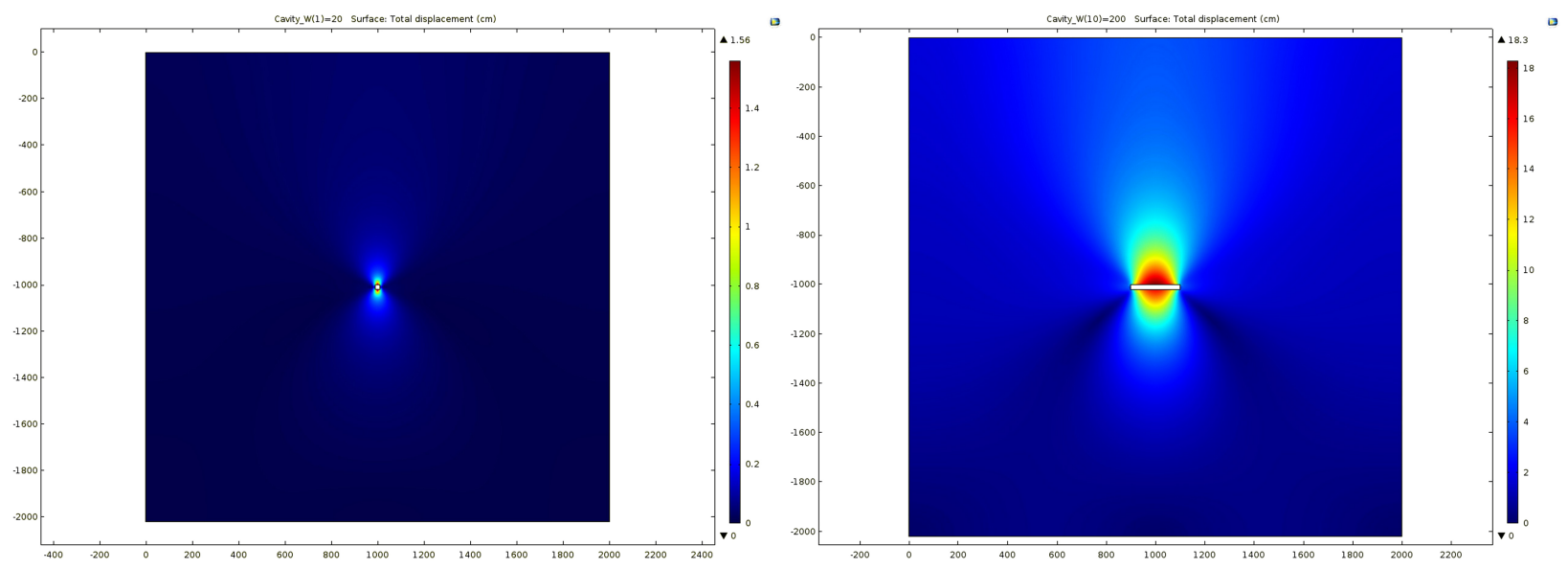

Figure 10. Total strata displacement around a deep seated cavity. 

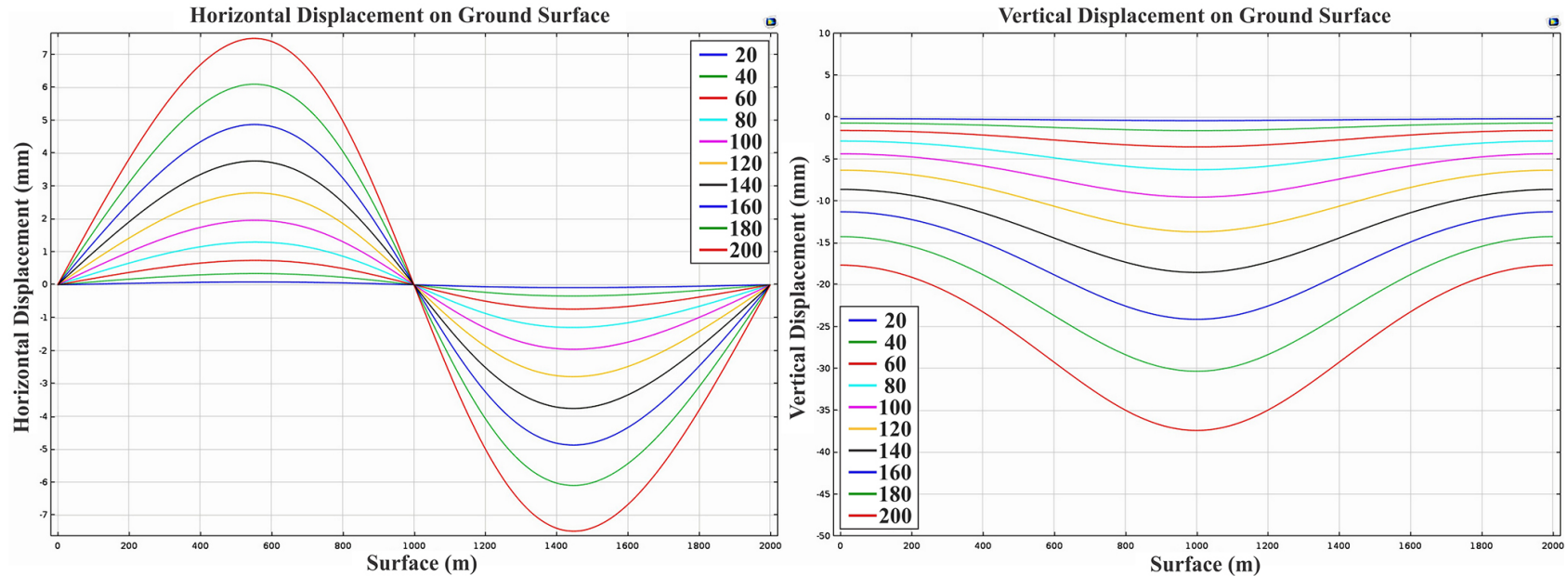

Figure 11. Horizontal and Vertical displacement of surface induced by a deep seated cavity.

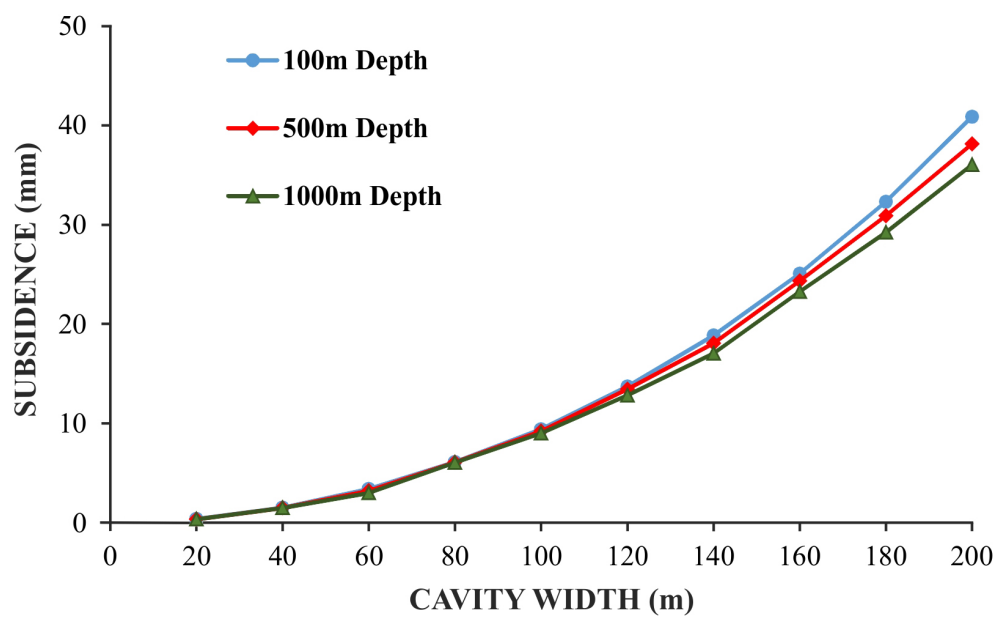

Figure 12. Effect of cavity width and depth on subsidence.
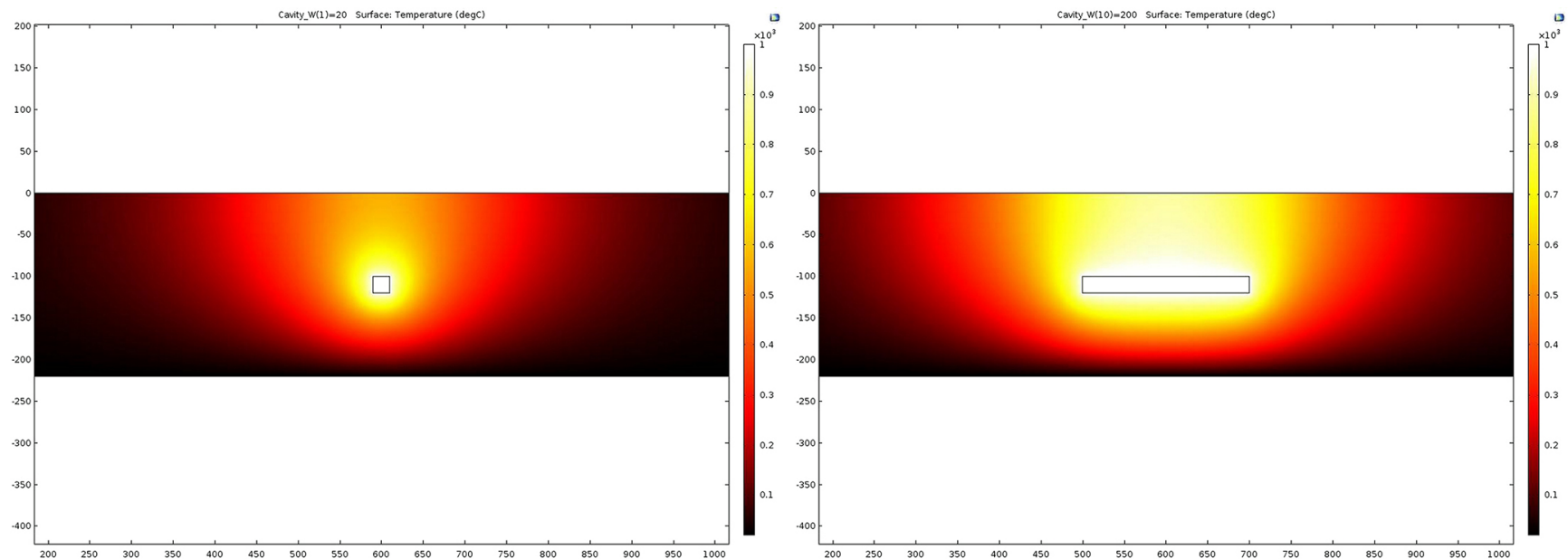

Figure 13. Temperature distribution around a shallow seated cavity. 

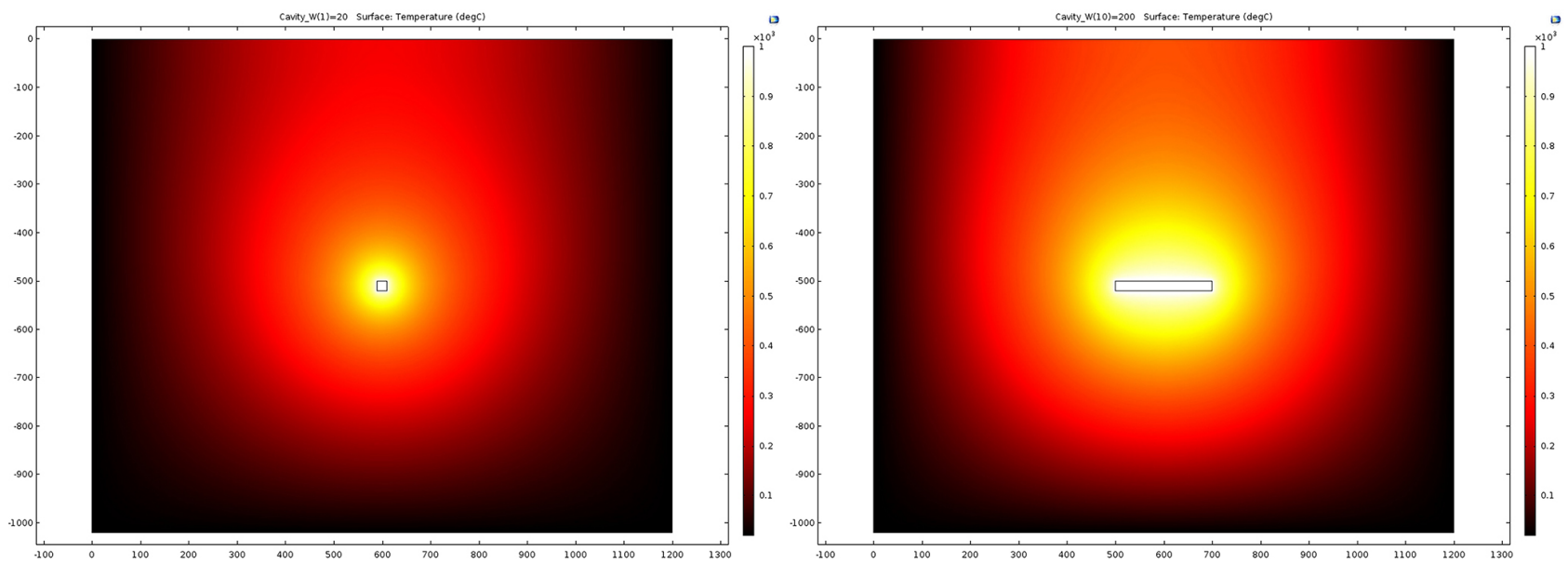

Figure 14. Temperature distribution around a medium seated cavity.
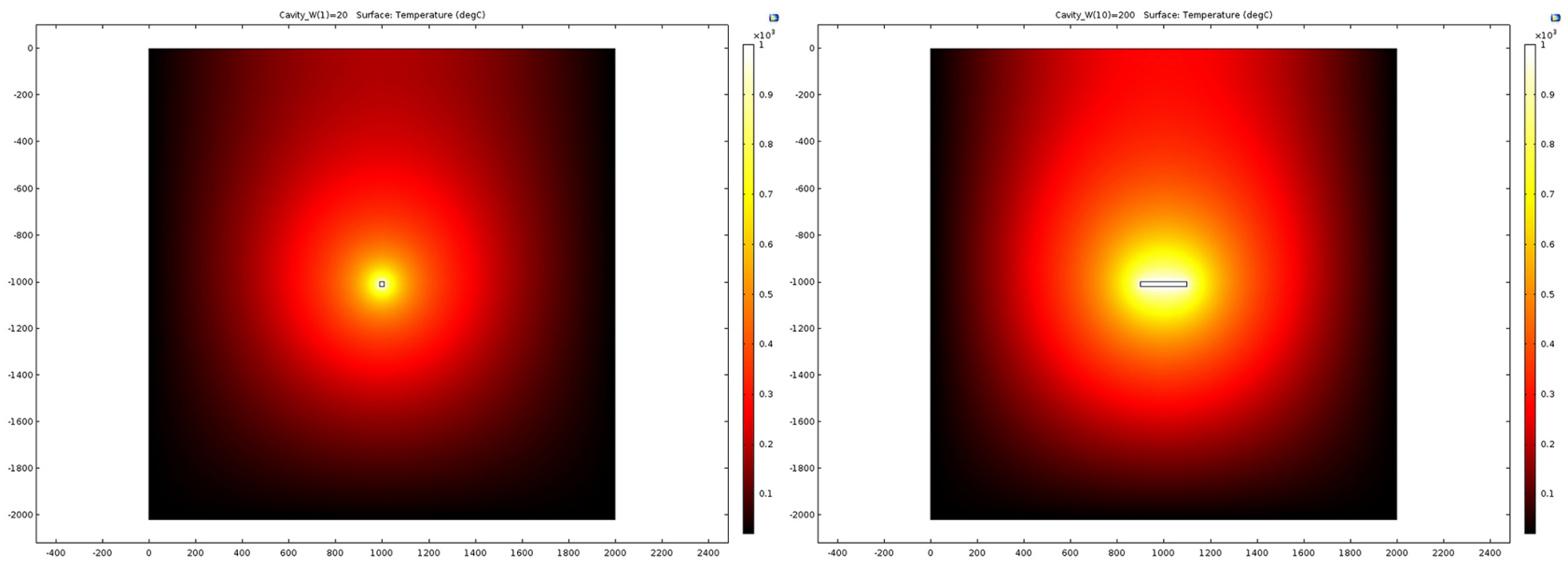

Figure 15. Temperature distribution around a deep seated cavity.

The amount of material required to fill in the $20 \mathrm{~m}$ cavity is far less than the $200 \mathrm{~m}$ cavity. A small opening thus causes less disturbance to the strata as compared to a large opening. The horizontal and the vertical displacements of the surface is shown in Fig. 7. Point 600 on the $x$ axis of both the graphs represent the midpoint of the cavity below the ground. A subsidence of approximately $40 \mathrm{~mm}$ is observed when the cavity width is $200 \mathrm{~m}$. Cavities created at a depth of $500 \mathrm{~m}$ induced less disturbance to the overlying strata as compared to the shallow seam model. The area just above the cavity displays the highest amount of displacement (Fig. 8). The effect of the cavity on the surface can be observed in Fig. 9. The surficial movements are less than those observed in shallow seam model. With the increase in the depth, the subsidence influence zone decreases. Maximum subsidence of $38 \mathrm{~mm}$ is observed when the cavity width is $200 \mathrm{~m}$. As the depth increases, the effect on the surface reduces. This can be seen in Fig. 10, where the cavity was created at a depth of $1000 \mathrm{~m}$. The effect on the surface is the least as compared to the shallow and the medium depth models (Fig. 11). The $200 \mathrm{~m}$ wide cavity induced the highest vertical displacement of $36 \mathrm{~mm}$. The graphs obtained for the horizontal displacement in Figs. 7, 9 and 11 follow the empirical model. A comparative analysis of vertical displacements obtained for cavities at various depths is shown in Fig. 12. It can be inferred that cavities having width below $120 \mathrm{~m}$ induce subsidence of similar magnitude. Only after $120 \mathrm{~m}$, do we observe a distinct variance in the magnitude of subsidence.

Variations in the temperature field around the cavities are shown in Figs. 13, 14 and 15. The region near the cavity are heated up to $1000^{\circ} \mathrm{C}$ which causes the rock to undergo several changes. These changes further affect the strength of the rocks. A shallow seated $(100 \mathrm{~m})$ UCG process generates a very high temperature zone over the ground surface as compared to medium $(500 \mathrm{~m})$ and deep seated $(1000 \mathrm{~m})$ processes. Figure 16 illustrates the temperatures observed for the point on the ground surface lying exactly over the centre of the cavity. 


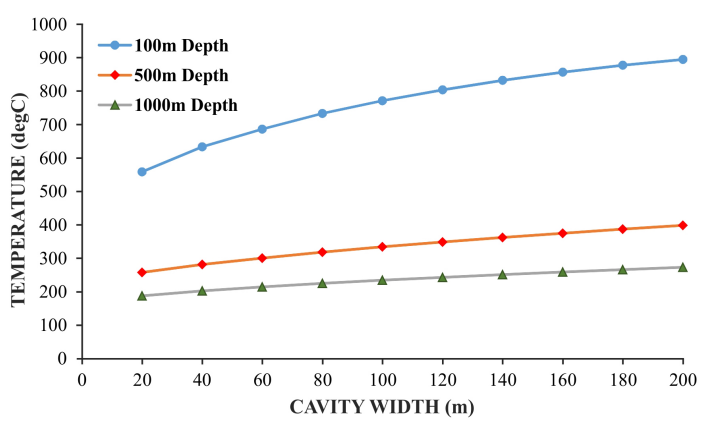

Figure 16. Effect of cavity width and depth on temperature.

\section{Conclusions}

The cavities less than $100 \mathrm{~m}$ in width induce low and similar magnitudes of subsidence on the surface. High magnitudes of subsidence are observed when the chamber is near to the ground surface. The magnitude of subsidence is inversely proportional to the depth at which the cavity is situated. The thermal studies of the cavity and the strata exhibit a similar relationship. It should be noted that the geomechanical and thermal studies were conducted for a homogenous strata consisting of single type of rock. The actual scenario observed in the field could vary owing to the presence of various strata and various geological discontinuities. The magnitude of subsidence and the heat distribution depends on the type of litho-units. Rocks such as siltstone, claystone and shale exhibit higher swelling properties as compared to sandstone. The presence of such rock types in the strata may lower the subsidence effect on the surface. Moreover, presence of ground water and geological discontinuities would affect the temperature distribution in the strata. Thus it is imperative to carry out a detailed geological exploration of the area before the commencement of UCG process. A cavity seated at a higher depth has minimal effect on the strata. Therefore, a deep seated coal seam is conducive for the application of UCG process.

Acknowledgements. The authors thank the anonymous reviewers for their careful review and contributions, which led to the improvement of the manuscript. The first author is thankful to the other three authors for their constant support and invaluable advice during the experimental and numerical analysis.

\section{References}

Bell, D. A., Towler, B. F., and Fan, M. H.: Coal gasification and its applications, William Andrew/Elsevier, Oxford, 101-111, 2011.

Burton, E., Friedmann, J., and Upadhye, R.: Best practices in Underground Coal Gasification, Lawrence Livermore National Laboratory, Livermore, California, 13-21, 2007.

Cena, R. J., Britten, J. A., and Thorsness, C. B.: Excavation of the partial seam CRIP underground coal gasification test site,
Proceedings of the 13th Annual Underground Coal Gasification Symposium, 24 August 1987, Laramie, Wyoming, USA, 1987.

Den'gina, N. I., Kazak, V. N., and Pristash, V. V.: Changes in rocks at high temperatures, J. Min. Sci., 29, 472-477, 1994.

Hajpál, M. and Török, Á.: Mineralogical and colour changes of quartz sandstones by heat, Environ. Geol., 46, 311-322, 2004.

Kratzsch, H.: Mining subsidence engineering, Springer-Verlag, Berlin, 30-31, 1983.

Luo, Y. G., Coertzen, M., and Dumble, S.: Comparison of UCG cavity growth with CFD model predictions, Proceedings of the 7th International Conference on CFD in the minerals and process industries, 9-11 December 2009, CSIRO, Melbourne, Australia, $1-5,2009$.

NCB (National Coal Board): Subsidence Engineers' Handbook, NCB Publications, Hobart House, London, 90-105, 1975.

Ranjith P. G., Viete, D. R., Chen, B. J., and Perera, M. S. A.: Transformation plasticity and the effect of temperature on the mechanical behaviour of Hawkesbury sandstone at atmospheric pressure, Eng. Geol., 151, 20-127, 2012.

Rao Q. H., Wang Z., Xie H. F., and Xie, Q.: Experimental study of mechanical properties of sandstone at high temperature, J. Cent. South Univ. T., 15, 478-483, 2007.

Reddish, D. J. and Whittaker, B. N.: Subsidence: Occurrence, Prediction and Control, Elsevier, Amsterdam, 359-376, 1989.

Shirsat, V. A.: Modeling of cavity growth in underground coal gasification, MS Thesis, Chemical engineering, Texas Tech University, 16-48, 1989.

Shirsat, V. A. and Riggs, J. B.: UCG cavity growth model, Proceedings of the 12th Annual Underground Coal Gasification Symposium, 21-28 August 1986, Saarbrucken, Germany, 297-304, 1986.

Somerton, W. H.: Thermal properties and temperature-related behavior of rock/fluid systems, Elsevier, Amsterdam, 148-166, 1992.

Tian, H.: Development of a Thermo-Mechanical Model for Rocks Exposed to High Temperatures during Underground Coal Gasification, PhD Thesis, Rheinisch-Westfälischen Technischen Hochschule (RWTH) Aachen University, Aachen, Germany, p. 10, 2013.

Tian, H., Kempka, T., Feinendegen, M., and Ziegler, M.: Study on ground subsidence development during and after underground coal gasification, EGU General Assembly, Vienna, Austria, 3-8 April 2011, EGU2011-1277-2, 2011.

Tian, H., Kempka, T., Neng-Xiong, X., and Ziegler, M.: Physical Properties of Sandstones After High Temperature Treatment, Rock Mech. Rock Eng., 45, 1113-1117, 2012.

Tian, H., Kempka, T., Yu, S., and Ziegler, M.: Mechanical Properties of Sandstones Exposed to High Temperature, Rock Mech. Rock Eng., Springer Verlag, Wien, 1-7, 2015.

Vishal, V., Pradhan, S. P., and Singh, T.: Tensile Strength of Rock Under Elevated Temperatures, Geotechnical and Geological Engineering, 29, 1127-1133, 2011.

Wu, G. and Liu, S.: Research on ultrasonic characteristics of sandstone after heating to high temperature, Boundaries of Rock Mechanics, Taylor \& Francis Group, London, 207-211, 2008.

You, L. J. and Kang, Y. L.: Effects of thermal treatment on physical property of tight rocks, Progress in Geophysics, 2015, 18501854, 2009. 